\title{
On the hadronic $\gamma$-ray emission from Tycho's Supernova Remnant $\dagger$
}

\author{
Xiao Zhang ${ }^{1}$, Yang Chen ${ }^{1,2}$, Hui $\mathbf{L i}^{3,1}$ and Xin Zhou ${ }^{4,2}$ \\ ${ }^{1}$ Department of Astronomy, Nanjing Univ., Nanjing 210093, China \\ ${ }^{2}$ Key Laboratory of Modern Astronomy and Astrophysics, Nanjing Univ., Ministry of \\ Education, China \\ ${ }^{3}$ Department of Astronomy, Univ. of Michigan, 500 Church Street, Ann Arbor, MI 48109, USA \\ ${ }^{4}$ Purple Mountain Observatory, 2 West Beijing Road, Nanjing 210008, China
}

\begin{abstract}
Tycho is one of nearly a dozen Galactic supernova remnants which are suggested to emit hadronic $\gamma$-ray emission. Among them, however, it is the only one in which the hadronic emission is proposed to arise from the interaction with low-density ambient medium. Based on the multi-band observations, we suggest that Tycho is encountering dense cloud at the northeastern boundary. The $\gamma$-ray emissions can be explained by hadronic process with self-consistent parameters, such as a modest energy conversion efficiency. In this SNR-cloud association scenario, the distance can be estimated as $\sim 2.5 \mathrm{kpc}$.
\end{abstract}

Keywords. supernova remnants, ISM: individual (Tycho), radiation mechanisms: non-thermal

\section{Introduction}

Hadronic $\gamma$-ray emission from supernova remnants (SNRs) is an important tool to test shock acceleration of cosmic ray (CR) protons. For Tycho's SNR which is suggested evolving in a tenuous medium, an energy conversion efficiency 10-15\% was invoked (e.g., Tang et al. 2011; Giordano et al. 2012; Morlino \& Caprioli 2012). Although it is suggested that Tycho is a naked Ia SNR without any dense cloud (Tian \& Leahy 2011), there seem to be signs and evidence in multiband showing the presence of dense clouds from the north to the east, which could be the target for bombardment of the accelerated protons and would thus reduce the needed converted energy. Constraints from $\gamma$-ray observation suggest that Cas A, another historical SNR, has only transferred a minor fraction ( $\leqslant$ $2 \%$ ) of the total kinetic energy to the accelerated particles (e.g., Araya \& Cui 2010). Moreover, under the Bohm limit assumption, the conversion factor no more than $1.3 \%$ is predicted in kinetic models for an SNR at the Tycho's age, evolving in a pre-shock medium of density $\sim 0.3 \mathrm{~cm}^{-3}$ (Berezhko \& Völk 1997). Therefore, the fraction of order $\sim 1 \%$ at Tycho's age deserves serious consideration.

\section{Hadronic Gamma-ray emission}

We assume that the energy spectrum of the accelerated particles (electrons and protons) is prescribed by a power law with high-energy cutoff. Some basic parameters are adopted as: distance $2.5 \mathrm{kpc}$, spectral index 2.3, explosion energy $6 \times 10^{50} \mathrm{erg}$ (Hughes 2000 ), the average downstream magnetic field $320 \mu \mathrm{G}$ and the energy conversion efficiency $\eta \sim 10^{-2}$. By fitting the radio- $\gamma$-ray data (see the left panel in Fig. 1), we get

$\dagger$ Supported by the 973 Program grant 2009CB824800, NSFC grant 11233001, grant 20120091110048 from the Educational Ministry of China, and grant 2011M500963 from the China Postdoctoral Science Foundation . 

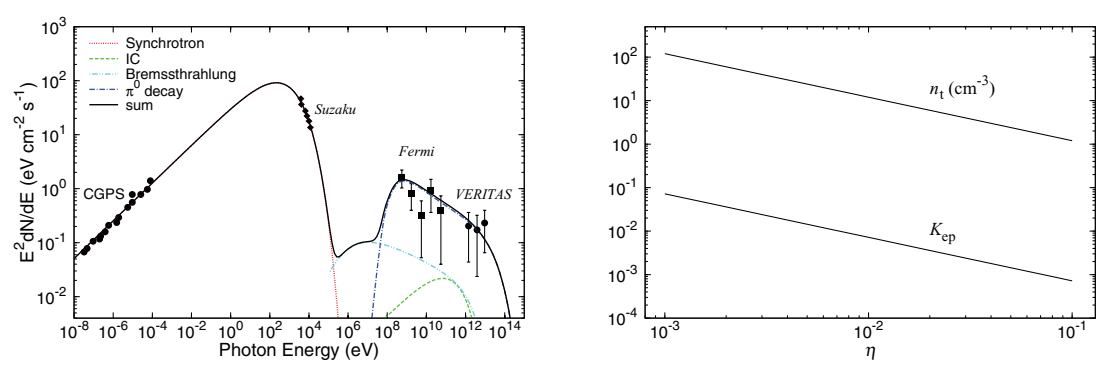

Figure 1. Left: Broadband SED of Tycho's SNR with the observed data in radio (Kothes et al. 2006), X-rays (Tamagawa et al. 2009) and $\gamma$-rays (Fermi: Giordano et al. 2012; VERITAS: Acciari et al. 2011). Right: Functional dependence of parameters $K_{\mathrm{ep}}$ and $n_{\mathrm{t}}$ on $\eta$.

the electron to proton number ratio $K_{\mathrm{ep}} \sim 0.7 \times 10^{-2}$ and the average density of the target protons $n_{\mathrm{t}} \sim 12 \mathrm{~cm}^{-3}$ (see the right panel in Fig. 1 for functional dependence of $n_{\mathrm{t}}$ and $K_{\mathrm{ep}}$ on $\eta$ ). Considering the shock compression ratio, the average pre-shock proton density is in the range $\sim 4-12 \mathrm{~cm}^{-3}$, which is much larger than the reported gas density $\sim 0.3 \mathrm{~cm}^{-3}$. This discrepancy can be reconciled if Tycho is encountering an intercloud medium. Indeed, there are some multi-band observational evidence of the presence of the surrounding dense medium from the north to the east: the expansion rate measured in Radio (Reynoso et al. 1997) and X-rays (Katsuda et al. 2010), CO-line observations (Lee et al. 2004; Cai et al. 2009; Xu et al. 2011), and infrared observations (Ishihara et al. 2010; Gomez et al. 2012).

The SNR-cloud association can be used to estimate the distance to Tycho's SNR. Due to the spiral shock, the local standard of rest velocity of the associated clouds allows two distances in two armed spiral shock model (Roberts 1972) for the longitude $120^{\circ}$. In combination with the line-of-sight HI absorption (Tian \& Leahy 2011), the distance is determined to be $\sim 2.5 \mathrm{kpc}$.

\section{References}

Acciari V. A., et al. 2011, ApJ(Letter), 730, L20

Araya M. \& Cui W., 2010, ApJ, 720, 20

Berezhko E. G. \& Völk H. J., 1997, Astroparticle Physics, 7, 183

Cai Z.-Y., Yang J., \& Lu D.-R., 2009, Chinese Astronomy and Astrophysics, 33, 393

Giordano F., et al. 2012, ApJ(Letter), 744, L2

Gomez H. L., et al. 2012, MNRAS, 420, 3557

Hughes J. P., 2000, ApJ(Letter), 545, L53

Ishihara D., et al. 2010, A\& A, 521, L61

Katsuda S., Petre R., Hughes J. P., Hwang U., Yamaguchi H., Hayato A., Mori K., \& Tsunemi H., 2010, ApJ, 709, 1387

Kothes R., Fedotov K., Foster T. J., \& Uyanıker B., 2006, A\&SA, 457, 1081

Lee J.-J., Koo B.-C., \& Tatematsu K., 2004, ApJ, 605, L113

Morlino G. \& Caprioli D., 2012, A\& A, 538, A81

Reynoso E. M., Moffett D. A., Goss W. M., Dubner G. M., Dickel J. R., Reynolds S. P., \& Giacani E. B., 1997, ApJ, 491, 816

Roberts W. W., 1972, ApJ, 173, 259

Tamagawa T., et al. 2009, PASJ, 61, 167

Tang Y.-Y., Fang J., \& Zhang L., 2011, Chinese Physics Letters, 28, 109501

Tian W. W. \& Leahy D. A., 2011, ApJ(Letter), 729, L15

Xu J.-L., Wang J.-J., \& Miller M., 2011, Research in Astronomy and Astrophysics, 11, 537 